\title{
BASES TEÓRICAS PARA UN ESTUDIO TRANSCULTURAL Y VARIACIONISTA DE LA (DES)CORTESÍA
}

\author{
THEORETICAL BASIS FOR A CROSS-CULTURAL \\ AND VARIATIONAL STUDY OF (IM)POLITENESS
}

\author{
Francisco Fernández García \\ Universidad de Jaén \\ Área de Lingüística General \\ fcofer@ujaen.es
}

Recibido: 21/01/2016

Aceptado: 29/03/2016

\begin{abstract}
Resumen
En el marco del imparable crecimiento de las investigaciones sobre la (des)cortesía durante las últimas décadas, este trabajo presenta una discusión teórica que se propone sentar las bases para la investigación de tal fenómeno desde un enfoque transcultural y variacionista. Con este fin, se discuten a) el papel de lo convencional y lo contextual en la configuración de la (des) cortesía, b) la incidencia de los factores de naturaleza cultural, social y situacional, c) el modo en que la conceptualización de lo (des)cortés como una conjunción de estrategias y mecanismos puede esclarecer su comprensión, y d) el marco teórico, en lo que a las teorías sobre la (des) cortesía respecta, más adecuado para el análisis.

PALABRAS CLAVE: (des)cortesía, análisis transcultural, análisis variacionista.
\end{abstract}

\begin{abstract}
Within the framework of the continuous growth of research on (im)politeness in recent decades, this paper presents a theoretical discussion that aims to lay the groundwork for the research of this phenomenon from a crosscultural and variational approach. To this end, several key issues are discussed, namely: a) the role of conventional and contextual elements in the shaping of (im)politeness; b) the impact that cultural, social and situational factors have; c) the way in which the conceptualization of (im) politeness as a combination of strategies and mechanisms can clarify its understanding; and d) the theoretical framework, regarding theories about (im)politeness, more suitable for our analysis.

KEYWORDS: (im)politeness, cross-cultural analysis, variational analysis.
\end{abstract}

Para citar este artículo / To cite this article: Fernández García, Francisco (2016). Bases teóricas para un estudio transcultural y variacionista de la (des)cortesía. ELUA, 30: 79-100. doi:10.14198/ELUA2016.30.04

Enlace / Link: http://dx.doi.org/10.14198/ELUA2016.30.04 


\section{Introducción}

Las investigaciones sobre la (des)cortesía ${ }^{1}$ lingüística vienen adquiriendo un imparable protagonismo en los estudios pragmático-discursivos desde hace décadas. Tras la aparición de las primeras propuestas al respecto allá por los años 70 y 80 del siglo pasado (con trabajos fundamentales como los de Lakoff, 1973; Brown y Levinson, 1978, 1987; y Leech, 1983), ha sido ingente el esfuerzo investigador dedicado a este campo, tal y como brevemente hemos explicado en otro lugar (Fernández García, 2014: 61-64). Es necesario destacar el papel nuclear que ha representado la aportación de Brown y Levinson (1978, 1987), que, a pesar de las numerosas críticas recibidas durante los años transcurridos desde su aparición, ha permanecido lustro tras lustro como referencia esencial de las investigaciones en este campo. Tales críticas han adoptado, esencialmente, dos direcciones, a saber,

a) la de quienes se han propuesto reformular el modelo original, modificando sus aspectos más discutibles y adaptándolo a los avances de la investigación en esta área (como Spencer-Oatey, 2002, 2008; o, en el ámbito hispánico, Bravo, 1999, 2003), y

b) la de quienes han optado negar la viabilidad de dicho modelo y formular un enfoque alternativo, entre los que destaca el conocido como "enfoque postmoderno" en el estudio de la cortesía (como Watts, 2003; o Locher y Watts, 2005).

El desarrollo de las investigaciones sobre la cortesía ha llevado aparejada, además, la aplicación de sus principios a un número creciente de géneros comunicativos, tanto del ámbito público como del privado, desde una perspectiva sincrónica pero también histórica, etc. Además, el interés exclusivo inicial sobre lo cortés ha ido dando paso a un protagonismo creciente del estudio de la descortesía. En este último campo, tras el trabajo pionero de Culpeper (1996), el volumen de publicaciones ha crecido exponencialmente en muy poco tiempo (con ejemplos representativos como los de Mills, 2005; Bousfield, 2008; o el mismo Culpeper, 2011a), hasta igualar, si no superar, el volumen de los dedicados al estudio de la cortesía.

El bagaje teórico y analítico acumulado en estas décadas ha elevado enormemente nuestro grado de comprensión acerca de los mecanismos que rigen la interacción social entre los individuos, de la que -es bien sabido- la interacción lingüística resulta un componente esencial. Y el aumento de dicha comprensión condujo pronto al planteamiento de tales cuestiones investigadoras desde una perspectiva intercultural y transcultural ${ }^{2}$, ante la percepción de que la complejidad de los aspectos relativos a la (des)cortesía involucrados en la interacción social aumenta enormemente cuando entre los hablantes median diferencias de origen cultural. El avance por este terreno ha conducido,

a) por un lado, al planteamiento de serias dudas acerca de la supuesta universalidad que reclamaban para sí los modelos pioneros (en trabajos como los de Matsumoto, 1988; Watts, 1992; o Bravo, 2004, entre otros) y,

1 Utilizaremos a lo largo de este trabajo los términos (des)cortesía y (des)cortés para hacer referencia conjunta a las facetas cortés y descortés de la comunicación, así como los términos cortesíaldescortesía y cortés/descortés para hacer referencia específica a cada uno de dichos ámbitos en particular.

2 Sobre las diferencias en el trabajo de ambas áreas de trabajo, véase, por ejemplo, Kádár y Haugh (2013: 242243). 
b) por otro, al desarrollo de importantes avances en el conocimiento de los desajustes que las diferencias culturales provocan en el modo en que los individuos conciben y gestionan la (des)cortesía (con trabajos como los de Wierzbicka, 2003; SpencerOatey, ed., 2008; o Kecskes, 2015, entre otros).

Ahora bien, ese interés por la comprensión de la vertiente intercultural de la (des)cortesía solo recientemente se ha visto acompañado por iniciativas encaminadas a la investigación del modo en que la variación en el comportamiento de este fenómeno toma cuerpo también en el plano intracultural e intralingüístico (como Schneider y Barron, eds., 2008, Schneider, 2012; o Cestero y Albelda, 2012).

Ciertamente, dicha dimensión adicional en el estudio de la (des)cortesía reviste un enorme interés, dado que, como señalan Mills (2011: 38) o Kádár y Haugh (2013: 45-47), las distintas sociedades (las distintas culturas, los distintos entornos culturales, etc.) distan de configurarse como bloques monolíticos y homogéneos, de manera que una caracterización global y sin matices de un determinado entorno cultural, a fuerza de generalizadora, acabará resultando engañosa (Mills y Kádár, 2011: 21-23). Por ello, para -tras huir del etnocentrismo- escapar también del sociocentrismo (Brenes Peña, 2011: 128), se hace cada vez más necesario indagar en tal variación intracultural si deseamos contar con una descripción realmente ajustada de la naturaleza del fenómeno analizado. Será, en este sentido, esencial encarar el análisis de lo (des)cortés conforme a la estratificación social de los hablantes, para, solo así, poder entender las diferencias interculturales de una manera dinámica, en la medida en que la caracterización de cada entorno cultural sea fruto de su comprensión como una suma de variedades.

Ahora bien, a esa comprensión dinámica del modo en que la cortesía se configura en las distintas sociedades le falta, conforme a lo descrito hasta aquí, un último factor, en absoluto de naturaleza menor: la comprensión de la variación situacional. Es decir, no solo variarán las manifestaciones de la (des)cortesía conforme lo hagan los entornos culturales, no solo lo harán en función de la estratificación social de los hablantes, sino que también, de manera manifiesta, lo harán conforme a la variación de los contextos de habla. Y es que no debemos pasar por alto que un determinado hablante, originario de una determinada cultura y marcado por unas determinadas variables sociolingüísticas, en función de los contextos de habla en los que se mueva, al igual que experimentará variaciones en distintos planos de su uso de la lengua (fónico, sintáctico, etc.), lo hará también en el modo de gestionar la interacción social y en el modo, por tanto, de percibir la (des)cortesía lingüística y comportarse respecto de ella.

Pues bien, a partir de todas estas premisas, estamos llevando a cabo un trabajo investigador que, sobre la base de un cuestionario presentado a informantes procedentes de dos distintos orígenes culturales (españoles en la ciudad de Jaén -Andalucía, España- e ingleses en la ciudad de Coventry -Midlands Occidentales, Reino Unido-), se marca como objetivo realizar una caracterización de ciertos aspectos clave en relación con la (des)cortesía lingüística, atendiendo a:

a) la variación cultural, es decir, la distinta procedencia lingüística y cultural de los hablantes,

b) la variación social, es decir, dentro de un mismo entorno lingüístico y cultural, la distinta estratificación social de los hablantes; y

c) la variación situacional, es decir, en todos los casos, los variados contextos comunicativos en los que el hablante se halle. 
Queda claro, no obstante, que el planteamiento habrá de ser de abajo hacia arriba, es decir, que si pueden obtenerse patrones culturales diferenciados habrá de ser a partir de la toma en consideración de la variación social y situacional; y, además, esos patrones culturales habrán necesariamente de ser entendidos como una suma de variedades y no como unas rígidas normas de carácter global, abarcadoras del conjunto del cuerpo social.

Como parte y requisito inexcusable de una investigación de tales características, el presente trabajo se centra en una discusión teórica acerca de cuatro cuestiones de importancia crucial al respecto, a saber:

a) el papel de lo convencional y lo contextual en el estudio de la (des)cortesía,

b) la incidencia en ella de los condicionantes culturales, sociales y situacionales,

c) la comprensión de la (des)cortesía como una conjunción de estrategias y mecanismos, y

d) el marco teórico idóneo, en lo que respecta a las teorías sobre la (des)cortesía, para encarar el análisis.

El objetivo esencial de este artículo, por tanto, es sentar las bases teóricas óptimas sobre las que poder edificar una investigación transcultural y variacionista de la (des)cortesía como la que proponemos, que nos permita avanzar en el conocimiento de este fenómeno de importancia capital para las investigaciones pragmático-discursivas actuales.

\section{Lo convencional y lo contextual}

A estas alturas de investigación sobre la (des)cortesía lingüística, casi cuatro décadas después del trabajo pionero de Brown y Levinson (1978), parece inexcusable asumir, con Brenes Peña (2011: 128), la inexistencia de "una relación biunívoca entre los elementos lingüísticos y las funciones sociales". Es decir, el ingente trabajo acumulado en estas décadas de investigación ha revelado que la relación entre los mecanismos lingüísticos utilizados por el hablante y el efecto (des)cortés de su enunciación dista de ser automática. En este sentido, resulta bastante plausible la idea (extendida entre los expertos en la materia, como, entre otros, Culpeper, 2011a: 125; Bousfiled, 2008: 55; Briz, 2004: 72; o Albelda y Barros, 2013: 23) de que una parte de los elementos ligados a lo (des)cortés tendrá una configuración esencialmente contextual mientras que otra parte tendrá una naturaleza principalmente convencional, de manera que la interpretación de la (des)cortesía oscilará entre ambos polos. Sin perder de vista, no obstante, que también hay opiniones, desde las posiciones más extremas del conocido como enfoque postmoderno en el estudio de la cortesía, que sostienen la imposibilidad de elaborar modelos predictivos a causa de omnipresente importancia de lo contextual (Watts, 2003: 23).

Briz (2012: 34), por ejemplo, explica que la cortesía, como actividad social de acercamiento al otro, y la atenuación, como estrategia lingüística (entre otras) para lograr dicho acercamiento, son pareja, pero son solo "una pareja de conveniencia"; esto es, la correlación atenuación-cortesía no es sistemática, dado que, pongamos por caso, los excesos de atenuación pueden, en determinados contextos, llegar a ser descorteses, o las ausencias de atenuación e incluso la presencia de intensificación, dependiendo de la situación de habla, no tienen por qué llevar aparejadas consecuencias descorteses. En consecuencia, si asumimos que el efecto (des)cortés de la enunciación de un hablante no es, frecuentemente, una 
consecuencia exclusiva y automática de los mecanismos lingüísticos utilizados, una comprensión acertada del fenómeno habrá de pasar, más que probablemente, por un enfoque discursivo de su análisis, es decir, por una percepción de la (des)cortesía como algo que no solo está contenido en palabras y oraciones sino que, en buena medida, conforme a la idea de los miembros del Linguistic Politeness Research Group (2011: 2), es también, y de manera muy importante, "a question of judgements made by participants and negotiated within talk". También Spencer-Oatey (2005: 97) incide en esta idea cuando señala que un determinado comportamiento no es, per se, cortés o descortés, sino que son los hablantes los que, conforme a su concepción de lo que es socialmente adecuado, catalogarán dicho comportamiento como (des)cortés. En una línea semejante, por citar una tercera opinión, se expresan Kádár y Haugh (2013: 43) cuando insisten en que no se puede prejuzgar a priori qué será lo (des)cortés, por ejemplo, en una determinada cultura, sino que hay que ir al discurso, al análisis, al contexto.

Los juicios de los hablantes dependerán de numerosas variables culturales, sociales y situacionales, circunstancia que hace fácilmente entendible el hecho de que una misma secuencia lingüística pueda llevar aparejados resultados manifiestamente distintos en el plano de la (des)cortesía según el contexto en que sea proferida. Y, además, no olvidemos que -según decíamos- lo (des)cortés es también algo que se negocia en la interacción, de manera que ni siquiera el umbral que separa lo cortés de lo descortés será algo necesariamente estable a lo largo de un determinado evento comunicativo, sin que ello quiera decir, en contrapartida, que los hablantes carezcan de convenciones generales que guíen su percepción al respecto.

Hemos de pensar, de este modo, conforme a la idea de Harris (Linguistic Politeness Research Group, 2011: 4-5), que no podemos fiar nuestro análisis a la pre-existencia de rígidas normas de cortesía, olvidando la incidencia discursiva de distintos marcos culturales y sociales, perspectivas ideológicas y convenciones y expectativas vinculadas a situaciones particulares. En definitiva, por tanto, parece claro que una investigación como la que encaramos, si pretende medir la variación de la percepción de lo (des)cortés en función de variables como las que mencionábamos más arriba, habrá de ser un trabajo de naturaleza contextual, discursiva, en la línea de lo expresado por el Linguistic Politeness Research Group (2011: 5):

That is, the focus is on what the language used means to the participants, including both speaker and hearer, whether the participants themselves classify the utterances as polite or impolite, how they come to make those judgements, and what information and cues inform those decisions about whether someone has been polite or impolite.

De hecho, al decir de la bibliografía especializada (por ejemplo, Kádár y Hauguh, 2013: 36), las investigaciones sobre la cortesía han experimentado en los últimos lustros, en general, un cambio metodológico en este sentido, hacia un análisis más discursivo e interaccional.

Pensemos, por ejemplo, en el análisis de lo (des)cortés vinculado a ciertos actos de habla, como el agradecimiento o el halago. Mills (2011: 21-22) señala que quedarse en un uso rígido de los principios clásicos de la teoría de los actos de habla (en la línea de Brown y Levinson, 1987) a la hora de analizar su función en el plano de la cortesía es insuficiente para un análisis certero de la variabilidad discursiva de tales acciones. Lo cual es cierto, sin duda: hay que tomar en consideración los contextos sociales y los marcos de habla; ahora bien, ello dista de 
significar que dichos actos carezcan de un cierto valor por defecto, convencional para los hablantes, en lo tocante a la (des)cortesía, por más que dicho potencial pueda ser no solo matizado, sino incluso anulado o revertido en un contexto de comunicación concreto. En este sentido, la apuesta por un enfoque discursivo no tiene por qué implicar necesariamente el rechazo de la teoría de los actos de habla, tal y como plantean ciertas posturas del enfoque postmoderno en el análisis de la cortesía (así lo explica, por ejemplo, Terkourafi, 2005: 241); al contrario, la teoría de los actos de habla puede ofrecer un soporte teórico idóneo sobre el que se aplique dicho análisis discursivo, que la dotará de unas mayores flexibilidad y adaptabilidad contextual.

De nuevo, por tanto, hablamos de la conveniencia de movernos, conforme a lo que apuntábamos con anterioridad, en la dialéctica entre lo convencional y lo contextual. En este sentido, y a propósito del enorme hincapié de los enfoques postmodernos en lo contextual, Ogiermann (2009: 18) critica que dichos enfoques ponen un excesivo énfasis en los potenciales desacuerdos entre hablante y oyente a la hora de interpretar qué sea (des)cortés, perdiendo de vista que ambos intercambian constantemente sus papeles y que interactúan en un entorno cultural común y conforme a unos patrones sociales compartidos. Efectivamente, sin perder de vista tales factores, habremos de pensar que la comprensión de la cortesía no puede quedarse en el análisis de expresiones u oraciones aisladas, habiendo de tener un enfoque más contextual, en la medida en que partimos de la base de que lo (des)cortés no es algo estrictamente inherente a las palabras usadas; pero, al mismo tiempo, que no todo es radicalmente contextual en el análisis de lo (des)cortés.

De este modo, por tanto, aunque aceptemos -con Mills (2011: 34-35) - la idea sobre la imposibilidad de establecer modelos predictivos universales a priori sobre la (des)cortesía, no solo podemos, sino que debemos esforzarnos por hallar patrones, tendencias, etc. en determinados contextos socioculturales y marcos situacionales. Es más, ese será, muy probablemente, el objetivo más productivo del trabajo investigador en este campo. La misma Mills (2011: 48-49), de hecho, lo reconoce de este modo más adelante, cuando escribe que "it is possible to make generalisations about politeness across language groups", y Terkourafi (2005: 245), por su parte, critica la "pesimista" postura postmoderna, dado que "one may note that prediction would seem to be constitutive of any theory", de manera que "the aim is to draw on situations experienced to make predictions about situations not (yet) experienced”. De manera semejante se expresa Schneider (2012: 1024-1025), quien critica expresamente a Watts (2003) por su postura acerca de la imposibilidad de hallar principios relativos a la percepción de lo (des)cortés por parte de los hablantes y, en sentido contrario, afirma que es posible "to determine intersubjectively what is considered appropriate in a given social situation in a particular community". Refirámonos por último, dentro de esta línea crítica, a Ogiermann (2009: 1), quien describe la evolución de los estudios sobre la cortesía hacia los enfoques postmodernos como un tránsito desde la pragmática hacia la teoría social, en el que cada vez se enfatiza más el carácter impredecible de lo (des)cortés.

Venimos señalando, pues, que lo (des)cortés oscila entre lo convencional y lo contextual; pero, al mismo tiempo, insistimos en que las formas lingüísticas no son intrínsecamente (des)corteses, dado que la incidencia de la situación comunicativa resulta clave en su funcionamiento. ¿Podemos, realmente, armonizar ambos planteamientos? ¿Podemos sostener que hay parte de convencional en la comunicación de lo (des)cortés mientras afirmamos que las formas lingüísticas no son, en sí mismas, corteses ni descorteses? Culpeper (2012: 1130) sugiere una idea interesante al respecto cuando señala que la clave puede estar en 
el hecho de que ciertas expresiones lingüísticas y ciertos contextos de comunicación han quedado convencionalmente unidos en determinados marcos comunicativos, de manera que, aunque su carga (des)cortés no sea algo intrínseco, sí que se trata de algo que, por defecto, viaja con ellas y es presupuesto por los hablantes. ${ }^{3}$ En este sentido podríamos entender, con Kaul de Marlangeon (2008: 254), que los hablantes posean, "como componente de su propia competencia comunicativa", una cierta tipología -adquirida en su comunidad de habla-para expresar y evaluar comportamientos (des)corteses.

Dejamos, no obstante, esta discusión abierta por el momento, para cerrarla más adelante, en el epígrafe 4, cuanto tratemos acerca de la distinción entre estrategias y mecanismos. Antes, hemos de detenernos en otra cuestión clave para nuestra investigación, también en relación directa con la de la convencionalidad/contextualidad, como es la naturaleza de los distintos tipos de condicionantes (culturales, sociales y situacionales) que inciden directamente en la percepción de la (des)cortesía por parte de los hablantes.

\section{Condicionantes culturales, sociales y situacionales}

La dialéctica entre lo convencional y lo situacional en el análisis de la (des)cortesía bien puede abordarse conforme a los planteamientos de Bourdieu (1991) a los que se refiere Mills (2011: 31): dentro de cada entorno social compartido, ciertas prácticas y actitudes sociales son percibidas como normales conforme a un determinado habitus, si bien esas prácticas son luego negociadas por los individuos, matizadas conforme a las circunstancias. Al papel del habitus habría que sumar la importancia de los marcos situacionales, de los que trata Terkourafi (2005: 247-248), explicando que ofrecen "an opportune way of representing situations holistically as structures of co-occurring components" (tales como la interacción entre ciertos tipos de hablantes - de cierta edad, sexo, clase social- entre los que median ciertos tipos de relaciones y que tiene lugar en ciertos tipos de entornos).

Holmes (2013: 376), por su parte, al hilo de sus explicaciones sobre el trabajo de la sociolingüística interaccional, incide igualmente en la importancia de los marcos para comprender la naturaleza de la interacción y los define de manera más amplia -incluyendo también el componente cultural- como "the set of expectations that we bring to an interaction, based on our previous experiences and our cultural norms". Incluso Kádár y Haugh (2013: 137), a pesar de su constante hincapié en la importancia de lo contextual y en la ineficacia de los modelos a priori para el análisis de la (des)cortesía, reconocen abiertamente la importancia de los marcos (schemata en su terminología), señalando que "reduce uncertainty in the information and interpretation of linguistic politeness for the simple reason that by relying on them the interactants can follow pre-existing ways of understanding politeness". Y ello es así porque, con frecuencia, de modo voluntario o no, los hablantes se sirven de formas y prácticas sociales habitualmente asociadas al marco en cuestión, aunque -claro está- no se trate de un proceso automático.

3 Si pensamos, por tanto, que el reconocimiento de lo (des)cortés descansa, en buena medida, en esos factores de recurrencia, es fácil entender, como explica Kecskes (2015: 45), que el funcionamiento de lo (des)cortés muestre significativos desajustes en la comunicación intercultural, dado que, en ella, "there is almost no reocurrence and no regularity of usage". De este modo, continúa Kecskes (2015: 46), parece ser que, en este tipo de intercambios comunicativos, cuanto menor es el dominio de las convenciones preexistentes por parte de los hablantes, mayor es el grado en que la interpretación de lo (des)cortés recae directamente sobre la literalidad semántica del enunciado. 
De este modo, cuando nos proponemos comprender de la manera más abarcadora posible la variación en la percepción que los hablantes poseen de la (des)cortesía, resulta muy clarificador entender que su funcionamiento se estructura en distintos niveles jerarquizados, es decir,

a) conforme a entornos culturales, esto es, en relación con los rasgos generales de una determinada sociedad;

b) conforme a grupos sociales, esto es, en relación con la estratificación social de los hablantes; $y$

c) conforme a marcos situacionales, esto es, en relación con los tipos de eventos de habla y el modo en que se desarrolle cada evento en cuestión.

En definitiva, por tanto, lo (des)cortés no habrá de ser visto como una mera elección individual del hablante, al estilo de Brown y Levinson (1987), sino como una elección inmersa en (y condicionada por) todos esos parámetros y presiones culturales, sociales y situacionales ${ }^{4}$.

En una línea semejante se expresa Bravo (2003: 104-105), cuando reconoce, al menos parcialmente, la existencia de esos distintos niveles en la configuración de la (des)cortesía: "hablamos de rasgos más o menos permanentes y reconocibles en la sociedad de origen, [...] los cuales no son estáticos, sino negociables en la interacción cotidiana". La idea de base, por tanto, es semejante a la que venimos manejando: cada cultura configura unos patrones en relación con la gestión de la (des)cortesía, pero dichos patrones no son algo rígido, sino algo así como una vía de cierta anchura por la que los hablantes pueden transitar, desplazándose hacia un lado u otro en función de las circunstancias de la interacción. En un trabajo previo, Bravo (1999: 164-165) hablaba también de una imagen básica, delimitada por componentes socio-culturales específicos, que luego se adapta a la situación de habla en función de los roles sociales de los hablantes, tanto permanentes (como los de género), como temporales (como los relativos a la edad) u otros que pueden permanecer o cambiar (como jefe / empleado, por ejemplo).

Albelda Marco (2008: 769), por su parte, cuando se dispone a analizar en qué medida los factores situacionales pueden condicionar la percepción de enunciaciones convencionalmente asociadas a lo (des)cortés, incide también en un acotamiento progresivo de los parámetros de configuración de la (des)cortesía, que arranca en el entorno cultural y acaba por cerrarse en la situación de habla:

Culture determines and restricts linguistic behaviours among speakers; the geographic variety of the language and culture enhance this process of restriction; the sociological variables limit the speakers' linguistic choices even further. Consequently, in a given communicative situation, the speaker will make use of those forms considered appropriate.

Debemos, pensar, por consiguiente, que los entornos culturales definirán unas primeras horquillas, unos primeros parámetros sobre lo que podrá (o tenderá a) ser considerado (des) cortés; dichos parámetros se verán acotados, en un segundo nivel de especificidad, al poner

4 Además de tales condicionantes, otro parámetro esencial es el temporal, tal y como analizan Kádár y Haugh (2013: 156 y ss.). No obstante, se trata de un factor que no contemplaremos aquí, dado que nos situamos en una perspectiva sincrónica y presente. 
en consideración los espacios sociales por los que deambulan individuos de determinada edad, sexo, nivel sociocultural, etc.; y, en el tercer nivel, se concretarán aún más conforme a los marcos comunicativos específicos en que se desarrolle la interacción y la manera en que esta vaya evolucionando.

Así lo vemos, por ejemplo, en las consideraciones de Briz (2012: 49-58) acerca de la variación en el uso de la atenuación. ${ }^{5}$ Partiendo de la clásica distinción entre culturas que tienden al acercamiento y culturas que tienden al distanciamiento (ejemplifica, precisamente, con la española vs. la inglesa), señala que la atenuación es más escasa en las primeras que en las segundas. Ahora bien, explica que estas tendencias generales varían en función de factores sociales y situacionales. Así, parece ser que, en la conversación española, los mayores tienden a atenuar más que los jóvenes, las mujeres más que los hombres y los hablantes de estratos sociales superiores más que los de estratos inferiores. Además, decíamos, en lo diafásico, la conversación española tiende a mostrar menor atenuación cuanto mayor es la igualdad entre los hablantes, cuanto mayor es la proximidad entre ellos y cuanto mayores son la cotidianidad y la informalidad de la conversación; y, al revés, mayor será la tendencia al uso de la atenuación cuanto menor sea el grado en que se den estos factores.

Resulta manifiesto, por consiguiente, que, cuando nos acercamos al estudio de la (des) cortesía, encaramos un trabajo investigador de naturaleza pragmática (más adelante entraremos en la disyuntiva entre pragmalingüística y sociopragmática ${ }^{6}$ ), con especial atención a la variación sociocultural y situacional. Y este campo de la variación pragmática nos coloca en un punto de confluencia entre la pragmática y la sociolingüística, dado que esta última disciplina sitúa entre sus objetivos esenciales explicar nuestro diferente uso del lenguaje en distintos contextos sociales (Holmes, 2013: 1). Dentro de la sociolingüística, a su vez, resultan pertinentes para nuestros intereses tanto su dimensión macro- como a su dimensión micro-. Ello es así en la medida en que, cuando nos planteamos el modo en que los individuos de distintas culturas interactúan mediante el lenguaje, conceptualizan a través de él sus respectivos mundos, etc., estamos adentrándonos en el terreno de la macrosociolingüística o sociología del lenguaje. Y, cuando, en segundo lugar, pretendemos analizar cómo la estratificación social, con las numerosas variables implicadas, afecta al funcionamiento del fenómeno estudiado, nos hallamos, manifiestamente, en el dominio de la microsociolingüística.

Desde este enfoque pragmático apoyado en la sociolingüística, vemos, pues, que el grado de convencionalidad existente en la percepción de lo (des)cortés por parte de los hablantes viene dado por los tres tipos de condicionantes (culturales, sociales y situacionales) analizados, que van acotando desde distintos ángulos el margen de variación en dicha percepción. Además, recordemos que cerrábamos el epígrafe anterior explicando que los individuos incorporan a su saber como hablantes todo un repertorio de recurrencias relativas a lo que acostumbra a funcionar como (des)cortés - añadamos ahora- en su entorno cultural, en los grupos sociales en que se mueven y en los marcos situacionales en que interactúan. Ahora bien, ¿podemos concretar más?, ¿podemos definir de manera más específica cómo se configura el papel de lo convencional y de lo situacional en la percepción de la (des)cortesía?

5 Vaya por delante -Briz (2012) insiste en ello a lo largo de su trabajo- que, como apuntábamos más arriba, la atenuación (como estrategia lingüística) y la cortesía (como fenómeno social), van con frecuencia de la mano, lo cual no implica que exista una correlación sistemática entre ellas: puede haber atenuación al margen de la cortesía y puede haber (des)cortesía con y sin atenuación.

6 Véase el epígrafe 4. 


\section{Estrategias y mecanismos}

En su trabajo dedicado a la presentación de las líneas maestras del enfoque discursivo en la investigación sobre la cortesía, Mills (2011: 38) insiste en la idea de que lo cortés y lo descortés no existen previamente al discurso, sino que dependen de lo que cuenta como tal para los hablantes en la conversación. Y, en este sentido, cita a Locher (2006: 734):

I deliberate refrain from labelling strategies such as boosting or hedging as more or less polite. [...] I do not wish to imply that I have already witnessed manifestations of politeness by simply identifying hedged utterances (or indirectness), nor that I have witnessed impoliteness by identifying unmitigated linguistic strategies (or directness).

Resulta acertada, efectivamente, la apreciación de Locher, tal y como muestra la experiencia analítica acumulada y como, por ejemplo, hemos discutido en diversos lugares (entre otros, Fernández García, 2015b) al analizar en qué medida lo implícito, lejos de actuar sistemáticamente en forma de mitigador, puede resultar un potenciador $-\mathrm{o}$, simplemente, el sostén esencial- del carácter atacante de un acto. También Kádár y Haugh (2013: 24-26), por ejemplo, insisten en que es un error establecer una correlación entre el uso de actos de habla indirectos y el carácter cortés de la enunciación. Spencer-Oatey (2005: 95-96), por su parte, señala que no intenta vincular estructuras lingüísticas concretas a la percepción de lo (des)cortés, dado que, en palabras de Fraser y Nolan (1981: 96), "no sentence is inherently polite or impolite".

Nos preguntamos, entonces, tal y como discutíamos más arriba, qué podemos saber a ciencia cierta sobre el valor (des)cortés de las enunciaciones. Pues bien, si la pregunta se plantea, por ejemplo, en los términos de si la intensificación de la enunciación es un procedimiento cortés o descortés, lo cierto es que no podemos obtener una respuesta definitiva, pues ambas funciones pueden ser desarrolladas por ella dependiendo de la configuración contextual de la enunciación (como explican, por ejemplo, Albelda y Barros, 2013: 41-44). ${ }^{7}$ La cuestión esencial en este sentido, a nuestro juicio, es que algo como la intensificación vocal, la ironía, el eufemismo o la interrupción, por citar algunos, no deben ser considerados estrategias ni de cortesía ni de descortesía, sencillamente porque ni una cosa ni otra son. Tal y como ampliamente hemos explicado en otros lugares (por ejemplo, en Fernández García, 2015a), el quid de la cuestión no estriba en el valor cortés o descortés de tales mecanismos, sino precisamente en el hecho de que deben ser considerados solo mecanismos, es decir, herramientas mediante las que podemos ejecutar nuestros actos lingüísticos de carácter tanto cortés como descortés.

Es cierto que tales mecanismos pueden tener, en función de esas asociaciones convencionales de las que hablábamos más arriba a partir de Culpeper (2012: 1130), ${ }^{8}$ un perfil principalmente orientado, en principio, hacia lo cortés o lo descortés, pero la asociación con una u otra función dista -lo hemos repetido ampliamente- de ser automática. Antes aludíamos, por ejemplo, a los mecanismos intensificadores, y fácilmente puede comprobarse cómo, en función de la variación situacional, mientras que en un contexto como el del deba-

7 Lo mismo ocurre, por poner un segundo ejemplo, con el mecanismo de la alo-repetición, como muestra el análisis de Bernal (2005).

8 Véase el epígrafe 2. 
te electoral cara a cara cumplen sistemáticamente una función descortés (Fernández García, 2015b), en la conversación cotidiana española parecen estar asociados de forma estable a actos de refuerzo de la imagen (Albelda, 2003). De este modo, hemos de pensar que los actos lingüísticos adquirirán su carácter (des)cortés, en el contexto en que sean proferidos, conforme a su orientación funcional, es decir, conforme a la estrategia nocional utilizada (o, dicho de otro modo, conforme al fin discursivo perseguido) por el hablante. Detengámonos en esta cuestión.

Un hablante puede pretender, pongamos por caso, mostrarse solidario con su interlocutor o, al contrario, ridiculizarlo, acciones sociales que sí poseen un perfil cortés o descortés definido, en la medida en que son evaluadas de forma recurrente por los hablantes en uno u otro sentido. E, incluso si aceptamos que el valor (des)cortés de tales estrategias discursivas no es, tampoco, universal (se puede insultar con una función cortés, por ejemplo9), podremos convenir en que dicho valor adquirirá configuraciones, como mínimo, previsibles dentro de determinados entornos culturales, grupos sociales y marcos comunicativos. En la ejecución de dichas estrategias, el uso de determinados mecanismos podrá poseer una importancia variable, desde ser un ingrediente más o menos accesorio hasta poseer una relevancia capital, pero difícilmente podremos afirmar que sea el mecanismo en sí el que posea un valor (des) cortés. Será solo la conjunción, en un contexto comunicativo determinado, de la estrategia funcional implementada y el mecanismo discursivo que la soporta la que propicie el surgimiento de dicha fuerza (des)cortés en la enunciación. ${ }^{10}$

Desde este enfoque, pues, los mecanismos serán, como decíamos, herramientas al servicio del hablante para cumplir su propósito funcional. En tal sentido, resulta revelador, por ejemplo, el trabajo de Culpeper (2011b) sobre la relevancia de la prosodia en el análisis de la descortesía. Culpeper (2011b: 57) señala que la ejecución oral del discurso puede ser clave para la interpretación del efecto descortés de un enunciado, no solo desambiguándolo, sino incluso dándole por completo la vuelta a su significado, como ocurre con frecuencia en la enunciación irónica. Y, del mismo modo, muestra (Culpeper, 2011b: 72 y ss.) la funcionalidad de los mecanismos prosódicos (velocidad, intensidad, pausas, etc.) para potenciar la descortesía en enunciados que ya resultan manifiestamente descorteses por su orientación funcional, esto es, por su mismo contenido. Los mecanismos, por tanto (en este caso, prosódicos), canalizan el propósito discursivo del hablante, a veces como refuerzo o punto de apoyo de un valor nocional definido y otras veces como un factor clave para la expresión de dicho valor nocional.

Hablamos, por tanto, de la comprensión del funcionamiento de lo (des)cortés como una conjunción de estrategias y mecanismos: estrategias que definen el propósito discursivo del hablante y mecanismos que canalizan la ejecución de dicho propósito. En relación con ello, Kádár y Haugh (2013: 26-28) describen los enfoques tradicionales de la cortesía (como toda la corriente brownlevinsoniana) como una combinación de formas y estrategias: formas (como un morfema, una palabra o una oración) convencionalmente asociadas a la cortesía

9 Asunto tratado, entre otros, por Zimmermann (2003).

10 A esta visión "estratégica" de la (des)cortesía podría oponérsele la objeción, conforme a los planteamientos de Cordisco (2005: 182-183), específicamente referidos a la descortesía, de que no siempre los actos del hablante obedecen a su intencionalidad, sino que pueden deberse, digamos, a diferentes "fallos de cálculo" en el contexto comunicativo. Reconociendo, desde luego, esta posibilidad como cierta, consideramos que se trata de un hecho más o menos marginal, en tanto en cuanto la comunicación humana posee una naturaleza manifiestamente intencional. 
y estrategias que actúan como "planes" orientados a obtener un fin. Nuestra concepción del análisis de lo (des)cortés como una conjunción de estrategias y mecanismos difiere, sin embargo, de dicho planteamiento en varios puntos esenciales:

a) en cuanto a la posible funcionalidad de los mecanismos, insistimos en que, aunque puedan rastrearse tendencias al respecto, no los entendemos vinculados a priori con lo cortés o lo descortés, sino como un soporte para la ejecución de las estrategias, estas sí, vinculadas a propósitos funcionales específicos;

b) en cuanto al repertorio de mecanismos utilizados, pensamos en un abanico mucho más amplio, no limitado a recursos léxicos o gramaticales, sino abarcador del conjunto de recursos de los que el hablante puede servirse para expresar sus objetivos funcionales, desde un ámbito local (como mecanismos gramaticales, léxicos o deícticos) a un ámbito discursivo (como mecanismos suprasegmentales, de planificación sintagmática o de estructuración y presentación de los contenidos) y un ámbito interaccional (como el uso de los pares de adyacencia o la función de las interrupciones);

c) en cuanto a la naturaleza de las estrategias, las definimos por su carácter estrictamente funcional, desvinculándolas netamente de los mecanismos $\mathrm{y}$, de este modo, purgándolas de las incoherencias que tradicionalmente se han arrastrado en su formulación al mezclarlas de modo más o menos indiscriminado con ciertos tipos de mecanismos; incoherencias que los mismos Kádár y Haugh (2013: 27) reproducen cuando mencionan, por ejemplo, "busca el acuerdo" junto con "utiliza metáforas", es decir, cuando colocan a un mismo nivel un recurso puramente nocional (es decir, una estrategia como "busca el acuerdo") y otro de naturaleza instrumental, vacío de contenido (es decir, un mecanismo como "utiliza metáforas") 11.

Hemos de añadir que esta nítida distinción entre estrategias y mecanismos, que define el alcance de lo convencional y lo contextual en nuestro análisis de la (des)cortesía, nos conduce, por otra parte, a dos parcelas claramente diferenciadas dentro del análisis pragmático:

a) a la vertiente pragmalingüística, es decir, al análisis de las formas lingüísticas que nos sirven para la ejecución discursiva de la (des)cortesía, una perspectiva analítica adoptada explícitamente, por ejemplo, por Briz (2003: 18), y

b) a la vertiente sociopragmática, es decir, al análisis de las funciones comunicativas desde una perspectiva más social, más volcada hacia el qué hacen (y no cómo lo hacen) los hablantes, enfoque claramente prevalente en trabajos como los de Bravo (1999 y 2005, por ejemplo).

Se trata de dos enfoques que aparecen abiertamente disociados en buena parte de las investigaciones sobre el tema, pero que poseen una naturaleza manifiestamente complementaria (siendo dos caras de una misma moneda), de manera que la realización de un análisis conjunto y global podrá ofrecer una óptima comprensión del fenómeno de la (des)cortesía y sus manifestaciones.

11 Para una breve discusión al respecto, véase Fernández García (2015b: 36-37). 
Llegados a este punto, nos queda un último aspecto por abordar para acabar de definir el armazón conceptual sobre el que se sustentará nuestra investigación transcultural y variacionista de la (des)cortesía, a saber, la definición misma de la cortesía y la descortesía, así como la elección del marco teórico adecuado para su análisis.

\section{La concepción de la (des)cortesía}

Conforme a todo lo explicado páginas atrás, parece claro que, en una investigación como la que proponemos, habremos de ir más allá de una concepción estrictamente individualista de la cortesía como la que se deriva del modelo de Brown y Levinson (1987), basada esencialmente en la idea de la mitigación del ataque hacia el interlocutor (Locher y Watts, 2005: 10). ${ }^{12}$ El papel de la (des)cortesía es algo mucho más complejo, no solo limitado al ámbito interpersonal hablante-oyente, sino imbricado en el contexto social, con el que interactúa. Mills (2011: 23-24) explica, por ejemplo, que el uso de fórmulas de deferencia no solo atañe al destinatario, sino también al reconocimiento y al mantenimiento del statu quo, del sistema. O pensemos también, como segundo ejemplo, en la cantidad de implicaciones grupales y sociales que lleva aparejado el uso del insulto ritual de solidaridad.

Bravo (2003: 103-104), por su parte, desde un punto de partida distinto, llega a un lugar semejante al del enfoque discursivo cuando, hablando del contexto sociocultural, explica que se trata de toda una serie de conocimientos compartidos por grupos de hablantes, que generan expectativas "acerca de lo que potencialmente puede ser evaluado como cortés, descortés o neutral en esa situación”. La misma Bravo (2003: 101-102), por otro lado, pone de manifiesto que las funciones que la cortesía ejerce en las relaciones interpersonales van mucho más allá de la mera mitigación del ataque contra la imagen del interlocutor y señala que "la cortesía es siempre una estrategia para quedar bien con el otro", tanto si es el objetivo primario de la enunciación (por ejemplo, cuando felicitamos o halagamos) como si persigue una función atenuadora (por ejemplo, cuando criticamos).

Manejamos, por consiguiente, una concepción social y contextual, así como también interaccional, de la (des)cortesía. Dicho carácter implica, por otro lado, que la investigación sobre lo (des)cortés pueda y deba servirse, complementariamente, de las herramientas metodológicas que ofrece el Análisis de la Conversación. De hecho, hace ya años que venimos defendiendo la pertinencia de la utilización conjunta de ambos marcos teóricos (véase, por ejemplo, Fernández García, 2000): en la medida en que la (des)cortesía es algo que los hablantes articulan en la interacción comunicativa, cuestiones como la configuración de los pares de adyacencia, la alternancia de turnos en el diálogo o el concepto de preferencia (una referencia esencial en este sentido es Schegloff, 2007) podrán resultar una pieza clave en su comprensión (como hemos mostrado en cuanto a su operatividad en la configuración del ataque descortés en el debate electoral: Fernández García, 2016).

En el marco discursivo y dialógico que venimos esbozando, ¿cómo podemos, pues, definir la cortesía y la descortesía? En cuanto a la cortesía, debemos empezar por señalar que nuestra apuesta por un enfoque discursivo e interaccional que supere la excesiva rigidez del

12 En este sentido, en más de una ocasión se ha puesto de relieve el carácter un tanto paranoico de la teoría, dada su fijación en la conflictividad inherente a la comunicación humana. Fijación, por cierto, que es llevada al paroxismo en trabajos como el de Gil (2006), para el que todo acto de habla es amenazante tanto para la imagen del hablante como para la imagen del oyente. 
modelo de Brown y Levinson (1987) no supone negar la validez del paradigma griceano, como ocurre desde las posturas postmodernas más extremas (por ejemplo, en Watts, 2003); al contrario, de lo que se trata es de dotar a dicho paradigma de unas mayores flexibilidad y potencia analítica. A partir de ahí, entre nuestros intereses fundamentales se hallan la elaboración de modelos sobre la (des)cortesía y el análisis de los mecanismos lingüísticos mediante los que se gestiona, lejos de esas posturas postmodernas más radicales que, como explica Grainger (2011: 171), parecen renunciar a ambos objetivos.

Por otro lado, en cuanto a la definición misma de qué es y qué no es un comportamiento comunicativo cortés, podemos comenzar por referirnos a la idea de Terkourafi (2005: 51) de que una aportación discursiva habrá de ser considerada cortés en la medida en que resulte una opción regular, esperable, en un determinado marco; conforme a dicha idea, por consiguiente, lo normal, lo que no provoque una reacción adversa del destinatario, sería lo cortés. No nos parece, sin embargo, que dicha propuesta acabe de estar justificada, resultando analíticamente más productivo distinguir:

a) aquellos comportamientos que pueden ser considerados corteses en la medida en que buscan mejorar la empatía, la relación social con el destinatario;

b) aquellos comportamientos que pueden ser considerados descorteses en la medida en que buscan el enfrentamiento, el deterioro de las relaciones con el destinatario; y, por último,

c) aquellos comportamientos que pueden considerados neutros, sin repercusiones reseñables respecto de la (des)cortesía.

De este modo, dentro de un determinado entorno cultural, hallaremos un abanico de comportamientos potencialmente corteses, descorteses y neutros, abanico que irá concretándose conforme a los condicionantes sociales y los marcos comunicativos específicos, es decir, conforme a la creciente especificidad de los tipos de situaciones de habla, de manera que, dentro de cada ámbito social, dentro de cada marco e, incluso, dentro del desarrollo de cada evento comunicativo, el punto neutro pueda ir variando; y, con él, el umbral de lo potencialmente cortés y descortés ${ }^{13}$.

Ahora bien, volvamos de nuevo un paso atrás. Asumiendo que un comportamiento comunicativo pueda resultar más o menos cortés, descortés o neutro en unos determinados contextos cultural y social y, específicamente, en un determinado marco comunicativo; asumiendo esto, necesitamos un esquema conceptual en el que entender en qué consiste realmente ese resultar o no más o menos (des)cortés. Partamos de la referencia inexcusable de Brown y Levinson (1987). Ya explicábamos en el epígrafe introductorio que han sido muchas, y de diversa índole, las críticas que ha recibido; no obstante, aun reconociendo que el modelo resulta criticable en distintos aspectos, consideramos que sus ideas sobre la gestión de la imagen siguen siendo un más que interesante punto de partida para el trabajo

13 Tanto el contemplar una zona intermedia de comportamientos neutros respecto de la (des)cortesía como el entender que dicha zona será susceptible de experimentar desplazamientos conforme a las características de la interacción son ideas que pueden hallarse, por ejemplo, en el trabajo de Bravo al que aludíamos más arriba (2003: 103-105). También en Spencer-Oatey (2005: 96-97) observamos que contempla en su modelo no solo lo (des)cortés, sino igualmente aquellos comportamientos que pueden tener repercusiones neutras respecto de la (des)cortesía. 
en este campo ${ }^{14}$, por más que deban ser reelaboradas para incorporar los avances logrados por la investigación desarrollada durante las décadas transcurridas desde su publicación. Entre las propuestas de reelaboración de sus principios, particular interés ofrece, en el ámbito hispánico, la realizada por Bravo (en trabajos como 1999, 2003 o 2008), con sus conceptos de imagen de autonomía e imagen de afiliación, la primera referida al deseo del individuo de ser visto por los otros como un elemento con identidad propia dentro del grupo y la segunda como su deseo de ser visto como un miembro identificado con el grupo (Bravo, 2008: 588). Se trata, como decimos, de un modelo muy interesante, que supera el excesivo individualismo de Brown y Levinson (1987), adquiriendo un carácter más social. No obstante, probablemente no resulte tan potente analíticamente como el configurado por Spencer-Oatey a lo largo de distintas publicaciones (2002, 2005, 2007, 2008).

Una virtud que la propuesta de Spencer-Oatey comparte con la de Bravo es precisamente el intento de superar ese individualismo del que veníamos hablando respecto de Brown y Levinson (1987). Así, Bravo (2003: 105) señala que, junto con la imagen individual, se puede distinguir una imagen de grupo (la familia, el partido político, el país, etc.). Además, queda claro que sus conceptos de imagen de autonomía e imagen de afiliación (Bravo, 1999: 160 y ss.) lo que hacen es precisamente entender la naturaleza del individuo en relación con el grupo social en el que se integra. Spencer-Oatey, por su parte, en su modelo de la gestión interrelacional (rapport management) pone el acento en la dimensión grupal y la relación interpersonal, como ella misma señala (Spencer-Oatey, 2002: 541). Devuelve el concepto de imagen a la idea original de Goffman (1967) y la descompone en distintos elementos que la hacen más potente analíticamente, precisamente en la medida en que distingue su dimensión individual de la grupal y la interaccional. A ello hay que añadir que desaparece el concepto de imagen negativa, muy criticado en la bibliografía especializada, siendo sustituido por el de derechos de socialización, más ajustado a su diferente naturaleza y capaz de recoger la diversidad de comportamientos y parámetros sociales existentes en distintas culturas. Por otra parte, resulta también interesante en el modelo de la gestión interrelacional el hecho de que, a diferencia de lo que hacían Brown y Levinson (1987), no pone el acento solamente en las estrategias encaminadas al mantenimiento de unas relaciones sociales armónicas, sin dar primacía a lo cortés ni a lo descortés (Spencer-Oatey, 2005: 96; Hernández López y Placencia, 2004: 131).

La fuerza que mueve el rapport management, explica Spencer-Oatey (2002: 540), posee, pues, dos componentes: la gestión de la imagen y la gestión de los derechos de socialización. La imagen "is concerned with people's sense of worth, dignity, honour, reputation, competence and so on" (Spencer-Oatey, 2008: 13) y tiene un carácter esencialmente afectivo, basado en el deseo del individuo de ser positivamente valorado por los demás. Se distinguen, conforme a análisis psicosociológicos de la identidad, la imagen cualitativa (quality face), relativa a un nivel estrictamente individual; la imagen identitaria (social identity face), relativa al nivel grupal, colectivo, es decir, atingente a la persona como miembro de un grupo o al grupo mismo; y la imagen relacional (relational face), concerniente al nivel

14 De hecho, como señalan Kádár y Haugh (2013: 2), por más que dicho paradigma haya sido criticado desde numerosos ángulos, lo cierto es que no se han llegado a proponer alternativas completamente consistentes. Ogiermann (2009: 20), por su parte, señala que los mayores logros de los enfoques postmodernos en el estudio de la cortesía residen precisamente en sus críticas al paradigma brownlevinsoniano, sin lograr, en contrapartida, ofrecer alternativas sólidas. 
interpersonal, esto es, a la persona en su relación con otra u otras personas relevantes, al rol social que representa y el modo en que gestiona la relación social con los demás. En cuanto a los derechos de socialización, "are concerned with social expectancies, and reflect people's concerns over fairness, consideration and behavioural appropriateness" (SpencerOatey, 2008: 13-14), esto es, lo que se considera que los demás deben hacer o no en ciertos contextos, ya sea conforme a una base regulada, ya sea conforme a una convención social. Son de dos tipos: los derechos de equidad (equity rights), que aluden al derecho del individuo a ser tratado con justicia, a no sufrir imposiciones u órdenes injustificadas, a no ser explotado, etc., y los derechos afiliativos (association rights), que conciernen al derecho del individuo a la relación social con otros y a ser tratado de modo acorde con el tipo y grado de dicha relación. Se trata, como decíamos, de un modelo muy interesante y de gran potencia analítica, que atiende a lo individual y a lo colectivo, al individuo en sí mismo y en sus relaciones con el grupo.

Este modelo de Spencer-Oatey será, por consiguiente, el armazón teórico sobre el que sustentemos nuestras apreciaciones sobre la percepción de la variación de la (des)cortesía por parte de los hablantes. Señalemos que, desde este punto de referencia, dejaremos al margen la distinción entre first order politeness y second order politeness sobre la que se viene insistiendo desde el enfoque postmoderno (Watts, 2003), entre otras cosas porque dista de ser evidente que los trabajos sobre la (des)cortesía hayan de estar necesariamente anclados junto a una u otra orilla. Así lo señala, por ejemplo, Schneider (2012: 1023), cuando escribe que "a reconciliation of these two positions does, however, seem possible". De hecho, la óptima comprensión del fenómeno vendrá, probablemente, de una perspectiva que combine un planteamiento teórico potente con una puesta en pie analítica que se halle enraizada en datos reales, en las percepciones de los hablantes.

El modelo de la gestión interrelacional, por el modo en que está planteado, no presupone una primacía de lo cortés o lo descortés, constituyendo un marco idóneo para el análisis de ambos planos de la interacción social entre los hablantes. No obstante, nos hemos centrado hasta aquí en este epígrafe principalmente en la consideración de lo cortés, de manera que prestaremos ahora atención específica, por último, a lo descortés. En este sentido, conviene distinguir lo que puedan ser ataques al interlocutor, por un lado, y un comportamiento descortés, por otro, en la medida en que un ataque (una crítica, por ejemplo) no necesariamente adoptará una configuración descortés, dado que puede aparecer acompañado de mecanismos mitigadores. Parece adecuada, en consecuencia, la definición de descortesía que ofrece Bousfield (2008: 72), para quien esta tiene lugar cuando el hablante, lejos de mitigar la fuerza de sus amenazas contra la imagen del interlocutor (añadamos, también contra sus derechos de socialización), las lleva a cabo de forma:

- i. Unmitigated, in contexts where mitigation is required, and/or,

- ii. With deliberate aggression, that is, with the face threat exacerbated, 'boosted', or maximised in some way to heighten the face damage inflicted.

Es decir, que, conforme a esta perspectiva, lo descortés hará su aparición cuando la amenaza contra el interlocutor adopte una dureza que este considere no acorde con las expectativas que la situación genera, ya sea por la ausencia de atenuación, ya sea por la presencia de intensificación. 
En esta línea, Culpeper (2011b: 59) ofrece una definición más detalla y con referencias más explícitas a los factores sociales, situacionales e interaccionales:

Impoliteness is a negative attitude towards specific behaviours occurring in specific contexts. It is sustained by expectations, desires and/or beliefs about social organisation, including, in particular, how one person's or a group's identities are mediated by others in interaction. Situated behaviours are viewed negatively -considered 'impolite'- when they conflict with how one expects them to be, how one wants them to be and/or how one thinks they ought to be. Such behaviours always have or are presumed to have emotional consequences for at least one participant, that is, they cause or are presumed to cause offence.

Resultará descortés, por consiguiente, aquel comportamiento comunicativo que deteriore la relación social entre los hablantes porque atente contra el equilibrio social que pueda esperarse en un determinado marco. Ello ocurrirá en tanto en cuanto dicho comportamiento dañe la imagen o los derechos de socialización del interlocutor sin que medie ningún tipo de mitigación o, un paso más allá, cuando se apoye en algún mecanismo potenciador de su agresividad.

En la medida en que hablamos de que lo descortés será aquello que tienda a provocar conflicto por dañar al interlocutor, habremos de incluir también aquellos intercambios comunicativos en los que se provoca tal daño aunque esa no fuera la intención comunicativa del hablante (algo en lo que insiste, por ejemplo, Kaul de Marlangeon, 2008: 258-259). Es decir, en la medida en que ponemos sobre la mesa la necesidad de un enfoque discursivo para la comprensión de la (des)cortesía, de ir al discurso para analizar, de facto, cómo los interlocutores evalúan lo (des)cortés, resulta manifiesto que el destinatario de un enunciado podrá, en función de innumerables condicionantes pragmáticos, percibir una carga descortés allá donde el hablante no tuvo intención de ponerla.

De hecho, circunstancias como esta vienen a redundar en la idea que venimos manejando acerca de los múltiples condicionantes que pueden incidir en la percepción de la (des)cortesía en un determinado intercambio comunicativo, lejos de rígidos patrones preestablecidos. Precisamente por ello, aunque propongamos la viabilidad de los parámetros culturales, así como, en un grado más de especificidad, la de los condicionantes sociales y situacionales, como guías generales para la percepción de lo (des)cortés, nos resulta difícil de aceptar que puedan proponerse escalas a priori sobre la gravedad de los actos de descortesía, aunque sea respecto de un marco cultural específico, como hace Kaul de Marlangeon (2008: 263) para la cultura hispánica:

1) Actos formalmente descorteses animados de un propósito cortés.

2) Actos descorteses involuntarios:

2.1. Metedura de pata.

2.2. Escatima involuntaria de la cortesía esperada por el oyente.

2.3. Prescindencia involuntaria de cortesía.

3) Autodescortesía.

4) Actos formalmente corteses animados de un propósito descortés.

5) Escatima deliberada de la cortesía esperada por el oyente.

6) Silencio abrumador.

7) Descortesía de fustigación. 
Sin entrar a analizar en detalle la escala, destaquemos, simplemente, que una clasificación como esta desprecia directamente el valor del contexto de habla. Es decir, nada justifica pensar que 2.2 resulte de antemano menos descortés que 5, en virtud, exclusivamente, de la presencia o ausencia de intencionalidad: cualquier hablante sabe, por propia experiencia, que las circunstancias de enunciación pueden llegar a hacer de 2.2 una agresión extremadamente dañina para el equilibrio de la relación social con el interlocutor, por más que el hablante no hubiera calculado tales consecuencias a la hora de proferir su enunciación ${ }^{15}$.

\section{Conclusiones}

Llegados a este punto, consideramos que nos hallamos en condiciones de encarar una investigación transcultural y variacionista de la (des)cortesía como la que proponíamos en la introducción. En el marco del imparable crecimiento de las investigaciones sobre la (des)cortesía en las últimas décadas, comenzábamos por destacar el interés de alcanzar una comprensión global del funcionamiento de los mecanismos atingentes a la gestión de la relación social entre los hablantes, una comprensión que, renunciando a aspiraciones de universalidad, integre los factores relativos a la especificidad cultural, social y situacional de los intercambios comunicativos. De este modo, planteamos un estudio de naturaleza transcultural, pero que, lejos de intentar caracterizar los distintos entornos culturales como bloques monolíticos, lo haga de forma dinámica, como una suma de variedades.

Con este objetivo, nos planteábamos en primer lugar, en el epígrafe 2, la discusión en torno a qué hay de convencional y qué de contextual en el funcionamiento de la (des) cortesía, aspecto esencial, claro está, a la hora de encarar un análisis transcultural. Esencial en la medida en que una concepción radicalmente contextual como la defendida por los enfoques posmodernos en el estudio de la (des)cortesía parece negar toda posibilidad de establecer generalizaciones al respecto y, con ello, de cualquier tipo de caracterización contrastiva entre distintos entornos culturales. En este sentido, llegábamos a la conclusión de que la comprensión de lo (des)cortés gravitará entre lo convencional y lo contextual, de manera que, aunque las formas lingüísticas no sean en sí mismas corteses o descorteses y la (des)cortesía sea algo que, en buena medida, "negocien" los hablantes en la interacción, esos mismos hablantes asumen convencionalmente la correlación entre ciertos componentes comunicativos y ciertas funciones sociales en el marco de contextos de habla específicos.

15 Hay otros problemas -digamos- de bulto en la propuesta de Kaul de Marlangeon (2008). Así, por referirnos a dos, en los puntos 1 y 6 de su escala. En cuando a 1 , el problema es bastante diáfano: no tiene sentido incluir en una escala de descortesía actos "animados de un propósito cortés", es decir, actos que, en virtud de las circunstancias pertinentes, busquen un efecto de cortesía, sean cuales sean sus características formales. En cuanto a 6 , Kaul de Marlangeon (2008: 261-262) habla del silencio abrumador como "único caso de descortesía ejercida por el oyente", cuando "esgrime deliberadamente su silencio para indicar desacuerdo con el hablante, a pesar de que el contexto de situación y el compromiso entre los interlocutores le impelirían a una respuesta concordante y a un encadenamiento preferido de aquiescencia”. Se trata, parece claro, de un error de percepción: el oyente no puede ser ni dejar de ser descortés; lo es en su condición de hablante que decide hacer uso de la palabra o comunicarse ostensivamente (Sperber y Wilson, 1986) por otros medios, como, en este caso, el silencio. 
Esa dialéctica entre lo convencional y lo contextual puede ser entendida satisfactoriamente, según planteábamos en el epígrafe 3, conforme a determinados condicionantes culturales, sociales y situacionales, que se superponen unos a otros en un grado creciente de especificidad. Es decir, existen, en primer término, condicionantes culturales que caracterizan, de una manera global, los patrones comunicativos de los hablantes. Ahora bien, resultaría engañoso quedarse ahí, sin reconocer que la estratificación social de los hablantes modulará de manera esencial la forma en que perciben y gestionan la (des)cortesía. Y, en tercer lugar, esos hablantes verán condicionada, una vez más, su manera de actuar respecto de la (des)cortesía según los marcos situacionales en los que se desarrollen los eventos comunicativos en los que se hallen envueltos. El modo en que los hablantes conciben y gestionan la (des)cortesía no será sino el resultado de la acción conjunta de esos tres tipos de condicionantes.

Partiendo de tales asunciones, en el epígrafe 4 cerrábamos la discusión sobre la dialéctica entre lo convencional y lo contextual con el establecimiento de la distinción clave entre estrategias y mecanismos. Se trata de un aspecto esencial para los objetivos de la investigación, dado que veíamos cómo las estrategias, de naturaleza nocional y netamente deslindadas de los mecanismos, adoptarán una configuración razonablemente estable y previsible dentro de determinados entornos culturales, grupos sociales y marcos situacionales, mientras que los mecanismos, de naturaleza lingüística y discursiva, aunque puedan tender (con un grado de convencionalidad muy variable) hacia lo cortés o lo descortés, tienen una naturaleza mucho más abierta e imprevisible.

Como último paso de nuestro planteamiento, abordábamos en el epígrafe 5 la discusión acerca del marco teórico adecuado para llevar adelante nuestra investigación transcultural y variacionista de la (des)cortesía. Reconociendo las carencias del modelo clásico de Brown y Levinson $(1978,1987)$ para un análisis que aspira a comprender la naturaleza social de la comunicación y que se interesa tanto por lo cortés como por lo descortés, abogábamos por un enfoque de naturaleza discursiva e interaccional como único camino mediante el que comprender la naturaleza real del funcionamiento de la (des)cortesía. Ahora bien, en lugar de negar el modelo y sus bases (la teoría griceana y la teoría de los actos de habla), defendíamos el interés de algunas propuestas teóricas que han reelaborado satisfactoriamente sus principios, logrando una mayor flexibilidad y potencia analítica. Y destacábamos, en este sentido, la propuesta de la gestión interrelacional de Spencer-Oatey $(2002,2008)$, que, entre otras ventajas, observa el fenómeno de la descortesía en su naturaleza social e interaccional y resulta adecuado tanto para el análisis de lo cortés como de lo descortés.

Cerrábamos el epígrafe introductorio señalando que el objetivo esencial de este trabajo era sentar las bases teóricas óptimas sobre las que poder edificar una investigación transcultural y variacionista de la (des)cortesía como la que proponíamos. En este sentido, tras la discusión acerca del papel de lo convencional y lo contextual en la percepción y la gestión de la (des)cortesía, en íntima conexión con la incidencia de los condicionantes culturales, sociales y situacionales, así como con la distinción entre estrategias y mecanismos; tras ello, y habiendo delimitado el marco teórico de la teoría de la gestión interrelacional como fructífero entorno de análisis, consideramos que, efectivamente, el objetivo se ha cumplido y nos hallamos en disposición de encarar con suficientes garantías un trabajo empírico que nos permita comprender un poco mejor cómo los hablantes interpretan y gestionan este aspecto esencial de la comunicación humana. 


\section{Referencias bibliográficas}

Albelda Marco, M. (2003): "Los actos de refuerzo de la imagen en la cortesía peninsular". En D. Bravo (ed.): Primer Coloquio del Programa EDICE. La perspectiva no etnocentrista de la cortesía: identidad sociocultural de las comunidades hispanohablantes. Estocolmo, Universidad de Estocolmo, págs. 298-305.

Albelda Marco, M. (2008): "Influence of situational factors in the codification and interpretation of impoliteness (Influencia de los factores situacionales en la codificación e interpretación de la descortesía)", Pragmatics, 18, págs. 751-773.

Albelda Marco, M., y M. J. Barros García (2013): La cortesía en la comunicación. Madrid, Arco/ Libros.

Bernal, M. (2005): "La alo-repetición como estrategia de cortesía y descortesía en la conversación conflictiva". En J. Murillo Medrano (ed.): Actas del II Coloquio del Programa EDICE. EstocolmoCosta Rica, Universidad de Estocolmo-Universidad de Costa Rica, págs. 55-79.

Bourdieu, P. (1991): Language and Symbolic Power. Cambridge, Polity Press.

Bousfield, D. (2008): Impoliteness in Interaction. Ámsterdam, John Benjamins.

Bravo, D. (1999): “¿Imagen positiva vs. imagen negativa? Pragmática socio-cultural y componentes de face", Oralia, 2, págs. 155-184.

Bravo, D. (2003): "Actividades de cortesía, imagen social y contextos socioculturales: una introducción”. En D. Bravo (ed.): Primer Coloquio del Programa EDICE. La perspectiva no etnocentrista de la cortesía: identidad sociocultural de las comunidades hispanohablantes. Estocolmo: Universidad de Estocolmo, págs.. 98-108.

Bravo, D. (2004): "Tensión entre universalidad y relatividad en las teorías de la cortesía". En D. Bravo y A. Briz (eds.): Pragmática sociocultural: estudios sobre el discurso de cortesía en español. Barcelona, Ariel, págs. 15-37.

Bravo, D. (2005): «Competencia en la pragmática sociocultural del español”. En J. Murillo Medrano (ed.): Actas del II Coloquio del Programa EDICE. Estocolmo-Costa Rica, Universidad de Estocolmo-Universidad de Costa Rica, págs. 363-374.

Bravo, D. (2008): «The implications of studying politeness in Spanish-speaking contexts: a discussion (Las implicaciones del estudio de la cortesía en contextos del español. (Una discusión)", Pragmatics, 18, págs. 577-603.

Brenes Peña, E. (2011): "Recursos lingüísticos al servicio de la (des)cortesía verbal. Los apéndices apelativos". En C. Fuentes Rodríguez, E. Alcaide Lara y E. Brenes Peña (eds.): Aproximaciones a la (des)cortesía verbal en español. Berna, Peter Lang, págs. 119-137.

Briz, A. (2003): "La estrategia atenuadora en la conversación cotidiana española". En D. Bravo (ed.): Primer Coloquio del Programa EDICE. La perspectiva no etnocentrista de la cortesía: identidad sociocultural de las comunidades hispanohablantes. Estocolmo, Universidad de Estocolmo, págs. $17-46$.

Briz, A. (2004): “Cortesía verbal codificada y cortesía verbal interpretada en la conversación”. En D. Bravo y A. Briz (eds.): Pragmática sociocultural: estudios sobre el discurso de cortesía en español. Barcelona, Ariel, págs. 67-93.

Briz, A. (2012): “La (no)atenuación y la (des)cortesía, lo lingüístico y lo social: ¿son pareja?”. En J. Escamilla Morales y G. Henry Vega (eds.): Miradas multidisciplinares a los fenómenos de cortesía y descortesía en el mundo hispánico. Barranquilla-Estocolmo, Universidad del AtlánticoPrograma Edice, págs. 33-75.

Brown, P., y S. C. Levinson (1978): "Universals in language usage: politeness phenomena". En E. N. Goody (ed.): Questions and Politeness: Strategies in Social Interaction. Cambridge, Cambridge University Press, págs. 56-311.

Brown, P., y S. C. Levinson (1987): Politeness. Some Universals in Language Use. Cambridge, Cambridge University Press. 
Cestero Mancera, A. M, y M. Albelda Marco (2012): "La atenuación lingüística como fenómeno variable", Oralia, 15, págs. 77-124.

Cordisco, A. (2005): "Subjetividad y conformación de interacciones descorteses". En J. Murillo Medrano (ed.): Actas del II Coloquio del Programa EDICE. Estocolmo-Costa Rica, Universidad de Estocolmo-Universidad de Costa Rica, págs. 181-207.

Culpeper, J. (1996): “Towards an anatomy of impoliteness”, Journal of Pragmatics, 25, págs. 349-367.

Culpeper, J. (2011a): Impoliteness. Using Language to Cause Offence. Cambridge, Cambridge University Press.

Culpeper, J. (2011b): “It's not what you said, it's how you said it': Prosody and impoliteness". En Linguistic Politeness Research Group: Discursive Approaches to Politeness. Berlín, De Gruyter, págs. 57-83.

Culpeper, J. (2012): “(Im)politeness: Three issues”, Journal of Pragmatics, 44, págs. 1128-1133.

Fernández García, F. (2000): Estrategas del diálogo. La interacción comunicativa en el discurso político-electoral. Granada, Método Ediciones.

Fernández García, F. (2014): "Impoliteness, pseudo-politeness, strategic politeness? On the nature of communicative behaviour in electoral debates", Círculo de lingüística aplicada a la comunicación, 58, págs. 60-89.

Fernández García, F. (2015a): "Si lee el programa y no lo entiende, tenemos un problema. Estrategias funcionales para el ataque descortés en el debate cara a cara", Cultura, lenguaje y representación / Culture, Language and Representation, 14, págs. 33-59.

Fernández García, F. (2015b): "El menosprecio y la burla como armas de ataque en el debate electoral. Caracterización funcional y configuración discursiva", Pragmática Sociocultural / Sociocultural Pragmatics, 3, págs. 32-58.

Fernández García, F. (2016): "Mecanismos interaccionales al servicio de la descortesía en el debate político", Spanish in Context, 13, págs. 263-284.

Gil, J. M. (2006): "Amenaza e invasión de la imagen. Un estudio sobre la naturaleza de la cortesía verbal”, Pragmalingüística, 14, págs. 75-86.

Goffman, E. (1967): Interaction Ritual. Nueva York, Anchor Books.

Grainger, K. (2011): “'First order' and 'second order' politeness: Institutional and intercultural contexts”. En Linguistic Politeness Research Group: Discursive Approaches to Politeness. Berlín, De Gruyter, págs. 167-188.

Hernández López, M., y M. E. Placencia (2004): "Modos de conducir las relaciones interpersonales en interacciones de atención al público: el caso de las farmacias en Sevilla y Londres", Estudios de Lingüistica. Universidad de Alicante, 18, págs. 129-150.

Holmes, J. (2013): An Introduction to Sociolinguistics ( $3^{\mathrm{a}}$ ed.). Harlow, Pearson.

Kádár, D. Z., y M. Haugh (2013): Understanding Politeness. Cambridge, Cambridge University Press.

Kaul de Marlangeon, S. (2008): "Tipología del comportamiento verbal descortés en español”. En A. Briz et alii (eds.): Cortesía y conversación: de lo escrito a lo oral. III Coloquio internacional del Programa Edice. Valencia, Universidad de Valencia y Programa Edice, págs. 254-266.

Kecskes, I. (2015): “Intercultural impoliteness”, Journal of Pragmatics, 86, págs. 43-47.

Lakoff, R. (1973): “The Logic of Politeness, or Minding your P's and Q's". En Proceedings of the Ninth Regional Meeting of the Chicago Linguistic Society, págs. 345-356.

Leech, G. N. (1983): Principles of Pragmatics. Londres, Longman

Linguistic Politeness Research Group (2011): "Introduction: The Linguistic Politeness Research Group". En Linguistic Politeness Research Group: Discursive Approaches to Politeness. Berlín, De Gruyter, págs. 1-17.

Locher, M. A. (2006): “The discursive approach to polite behavior: a response to Glick", Language in Society, 35, págs. 733-735.

Locher, M. A., y R. J. Watts (2005): "Politeness Theory and Relational Work", Journal of Politeness Research, 1, págs. 9-33. 
Matsumoto, Y. (1988): "Reexamination of the universality of face: politeness phenomena in Japanese", Journal of Pragmatics, 12, págs. 403-426.

Mills, S. (2005): "Gender and impoliteness", Journal of Politeness Research, 1, págs. 263-280.

Mills, S. (2011): "Discursive approaches to politeness and impoliteness". En Linguistic Politeness Research Group: Discursive Approaches to Politeness. Berlín, De Gruyter, págs. 19-56.

Mills, S., y D. Z. Kádár (2011): "Politeness and culture”. En D. Z. Kádár y S. Mills (eds.): Politeness in East Asia. Cambridge, Cambridge University Press, págs. 21-44.

Ogiermann, E. (2009): On Apologising in Negative and Positive Politeness Cultures. Ámsterdam, John Benjamins.

Schegloff, E. A. (2007): Sequence Organization in Interaction. Cambridge, Cambridge University Press.

Schneider, K. P. (2012): “Appropriate behaviour across varieties of English”, Journal of Pragmatics, 44, págs. 1022-1037.

Schneider, K., y A. Barron (eds.) (2008): Variational Pragmatics: A Focus on Regional Varieties in Pluricentric Languages. Ámsterdam, John Benjamins.

Spencer-Oatey, H. (2002): "Managing rapport in talk: Using rapport sensitive incidents to explore the motivational concerns underlying the management of relations", Journal of Pragmatics, 34, págs. 529-545.

Spencer-Oatey, H. (2005): “( $\mathrm{Im})$ Politeness, face and perceptions of rapport: unpackaging their bases and interrelationships", Journal of Politeness Research, 1, págs. 95-119.

Spencer-Oatey, H. (2007): "Theories of identity and the analysis of face", Journal of Pragmatics, 39, págs. 639-656.

Spencer-Oatey, H. (2008): "Face, (Im)Politeness and Rapport". En H. Spencer-Oatey (ed.): Culturally Speaking. Culture, Communication and Politeness Theory. Londres, Continuum, págs. 11-47.

Spencer-Oatey, H. (ed.) (2008): Culturally Speaking. Culture, Communication and Politeness Theory (2nd edition). Londres, Continuum.

Sperber, D., y D. Wilson (1986): Relevance: Communication and Cognition. Londres, Blackwell.

Terkourafi, M. (2005): "Beyond the micro-level in politeness research", Journal of Politeness Research, 1, págs. 237-262.

Watts, R. (1992): "Linguistic politeness and politic verbal behavior: reconsidering claims for universality”. En R. Watts, S. Ide y K. Ehlich (eds.): Politeness in Language: Studies in his History, Theory and Practice. Berlín, Mouton de Gruyter, págs. 43-69.

Watts, R. J. (2003): Politeness. Cambridge, Cambridge University Press.

Wierzbicka, A. (2003): Cross-Cultural Pragmatics. The Semantics of Human Interaction (2. ${ }^{\text {a }}$ ed.). Berlín, Mouton de Gruyter.

Zimmermann, K. (2003): "Constitución de la identidad y anticortesía verbal entre jóvenes masculinos hablantes de español”. En D. Bravo (ed.): Actas del Primer Coloquio del Programa Edice. Estocolmo, Universidad de Estocolmo, págs. 47-59. 